\title{
Classification of Skin cancer using deep learning, ConvolutionalNeural Networks - Opportunities and vulnerabilities- A systematic Review
}

\author{
Ravi Manne* $*^{1,2} \mid$ Snigdha Kantheti ${ }^{3} \mid$ Sneha Kantheti ${ }^{4}$ \\ ${ }^{1}$ Chemtex Inc, Texas,USA, Email: Ravimannemr@gmail.com \\ 2Master's In pharmacy, Texas, USA \\ ${ }^{3}$ Bachelor of Medicine, Bachelor of Surgery at NRI Medical college, Mangalagiri, India \\ ${ }^{4}$ Software Developer, Texas- USA
}

\section{To Cite this Article}

Ravi Manne, Snigdha Kantheti and Sneha Kantheti, "Classification of Skin cancer using deep learning, ConvolutionalNeural Networks - Opportunities and vulnerabilities- A systematic Review”, International Journal for Modern Trends in Science and Technology, 6(11): 101-108, 2020.

\section{Article Info}

Received on 16-October-2020, Revised on 11-November-2020, Accepted on 13-November-2020, Published on 18-November-2020.

\section{ABSTRACT}

Background: Skin cancer classificationusing convolutional neural networks (CNNs) proved better results in classifying skin lesions compared with dermatologists which is lifesaving in terms of diagnosing. This will help people diagnosetheir cancer on their own by just installing app on mobile devices. It is estimated that 6.3 billion people will use the subscriptions by the end of year 2021[28] for diagnosing their skin cancer.

Objective: This study represents review of many research articles on classifying skin lesions using CNNs. With the recent enhancement in machine learning algorithms, misclassification rate of skin lesions has reduced compared to a dermatologist classifying them.In this article we discuss how using CNNs has evolved in successfully classifying skin cancer type, and methods implemented, and the success rate. Even though Deep learning using CNN has advantages compared to a dermatologist, it also has some vulnerabilities, in terms of misclassifying images under some Criteria, and situations. We also discuss about those Vulnerabilities in this review study.

Methods: We searched theScienceDirect, PubMed,Elsevier, Web of Science databases and Google Scholar for original research articles that are published. We selected papers that have sufficient data and information regarding their research, and we created a review on their approaches and methods they have used. From the articles we searched online So far no review paper has discussed both opportunities and vulnerabilities that existed in skin cancer classification using deep learning.

Conclusions: The improvements in machine learning, Deep learning techniques, can avoid human mistakes that could be possible in misclassifying and diagnosing the disease. We will discuss, how Deep learning using CNN helped us and its vulnerabilities.

Keywords: Skin cancer,Deep Learning, Skin cancer classification, CNN, Transfer Learning, Machine Learning.

\section{INTRODUCTION}

Skin cancer is one of the most dangerous types of cancers which is caused by abnormal multiplication of cells. There are threemain types of skin cells are: Squamous, Basal and Melanocytes. Melanocytes is the most dangerous compared to 
other two types. It is again classified into Melanocytic and non-Melanocytic. It is exceedingly difficult to differentiate between malignant and benign melanoma, so dermatologists sometimes misclassify the malignant and benign melanoma. Melanoma is 19th most frequent cancer, and the number of annual cases has increased by $53 \%$,some reason is because of UV exposure[1,2].It is riskier than the Squamous and Basalas it spreads throughout the body faster. So, it is very crucial to identify or classify the correct type of cancer in early phases, to lower the death risk.

In the process of diagnosing the malignant lesion, dermatologist would do a visual inspection of the infected area, as a preliminary step.Many times, this would lead to wrong detection especially when the cancer is at early stages. Some lesion types are similar to each other, so correct diagnosis as required inorder to avoid complications. With just visual inspection of a dermatologist the accuracy rate of a dermatologist used to be $65 \%-80 \%$ [3].Dermatologists rarely achieve more than $80 \%$ sensitivities[15]In case of suspicious lesionalong with the visual inspection, dermatoscopicimages were taken with a special high resolution and magnifying camera lens.During this process of recording, the effect of light on the image is controlled by using filters, by reducing reflections in the skin, and there by making deeper skin layers visible.With this extra technical support, the accuracy rate has increased by additional 49\%[4]. and total rate of accuracy for a dermatologist visual check followed by dermoscopic images is of $75 \%-84 \%[5,6]$.

With the complications related to diagnosis using human eye, this is where Computer vision can help in diagnosing skin cancer.We can diagnose and classify skin cancer by traditional Machine Learning or by Deep Learning. In traditional Machine Learning technique, a domain expert needs to identify the applied features and make them more clearly visible to leaning algorithm to work, in order to reduce the complexity.Deep Learning algorithm, is a subfield of Machine Learning, works on artificial neural networks, which are algorithms inspired by the structure and function of the brain. ANN(Artificial Neural Network) can be trained on numerous number of images of both benign and malignant. By learning the nonlinearinteractions, the model itself can tell itself if the image is malignant or benign.So, in deep learning there is no need of domain expertise forfeature extraction. In this study, we will focus on deep learning using CNNs
(Convolutional Neural Networks), which is a type of ANNs.

\section{Convolutional Neural Networks}

CNNs are a kind of Artificial Neural networks which were proven to be immensely powerful in areas such as image recognition and classification [7]. Every ANN has at least 3 layers: an input layer which takes the input dataset that was fed, a hidden layer trained on the inputs fed to the input layer, and an output layer, depending on the input fed it gives the output.CNNs are trainedusing labeled dataset given with the respective classes.CNNs learn the relationship between class labels and the input objects comprise two components, one is the hidden layer which extracts the features and, the fully connected layers that does the classification task.

Figure 1:

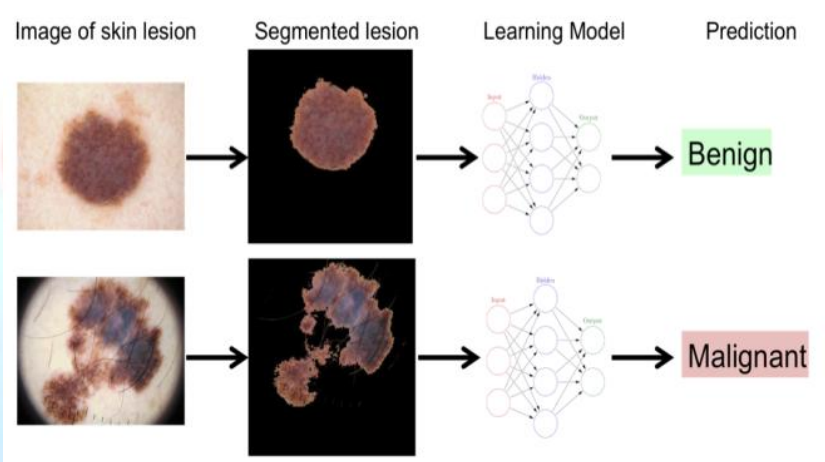

CNNs are used to classify skin lesions in two different ways. One is, a CNN can be applied as a feature extractor which is pretrained on large dataset, such as ImageNet [8]. In this case, classification is performed by another classifier, likesupport artificial neural networks, vectormachines, or k-nearest neighbors. Second one is, using end-to-end learning, a CNN can directly learn the relationship between the raw pixel data and class labels. Unlike the workflow used in machine learning, we do not need human expertise for feature extraction, it is no longer considered as independent step as it is now an integral part of classification step.The CNN trained by end-to-end learning, the processis again divided into two different types: learning from scratch or transfer learning.

\section{METHODS}

\subsection{Strategy}

We searched Google scholar, Elsevier, Pub Med, Research gate and took some of the valuable research works on skin classification using deep 
learning, using Convolutional neural networks, and we did a review on the performance metrics, and findings on how using deep learning in skin cancer classification has improved accuracy on classifying skin cancer compared to a dermatologist.

\subsection{Performance Metrics:}

Model Evaluation is performed by below following metrics [30].

Specificity: It gives what fraction of all negative classes are correctly identified as negative my model classifier. Also called True Negative Rate.

\section{$\mathbf{S P}=\mathbf{T N} /(\mathbf{T N}+\mathbf{F P})$}

Sensitivity: It is the ability of the process to correctly identify the disease condition or situation.

$$
\mathbf{S E}=\mathbf{T P} /(\mathbf{T P}+\mathbf{F N})
$$

ROC AUC: Area under receiver operating characteristic. It is the probability that the classifier will identify TPR against FPR. It is the graph between true positive rate vs false positive rate.

Precision: It gives what fraction of all classes that are correctly predicted positive, are actually positive.

\section{PREC = TP / (TP + FP)}

Negative Predictive Value (NPV): It is the probability of the inputs that tested negative, truly does not have the disease.

\section{NPV $=$ TN / (TN + FN)}

Positive Predictive Value (PPV): It is the probability of the classes that are tested positive, truly have the disease.

\section{$\mathbf{P P V}=\mathbf{T P} /(\mathbf{T P}+\mathbf{F P})$}

Dice coefficient: It gives the overlap measure between the automatic and the ground truth segmentation. It is also called as overlap index.

\section{DC $=2$ TP $/(2 . T P+F N+F P)$}

\section{Where}

- True negative (TN): negative class is correctly predicted by the classifier model

- True positive (TP): positive class is correctly predicted by the classifier model

- False negative (FN): negative class is incorrectly predicted by the classifier model

- False positive (FP): positive class is incorrectly predicted by the classifier model.

\section{SKIN LESIONS CLASSIFICATION BASED ON CONVOLUTION NEURAL NETWORKS: OPPORTUNITIES}

4.1. Skin Lesion Classification Using Convolutional Neural Network as a Feature Extractor

[9]The author has used U-net algorithm of CNN for segmentation process. They used, Edge Histogram
(EH), Local Binary Pattern (LBP), Gabor method and Histogram of Oriented Gradients (HOG), to extract the features fromthe segmented images. Features that are extracted from the above mentioned methods were fed into the Support Vector Machine (SVM), and also K-Nearest Neighbor (KNN), Naïve Bayes (NB) and Random Forest (RF)classifiers, to diagnose whether it is benign or Melanoma.This experiment is carried out with 900 dermoscopic images.International Skin Imaging Collaboration (ISIC) is used for images $.10 \%$ of the 900 segmented images areused as test data and the remaining $90 \%$ of the 900 images are used as training data for classification

These features were fed into the classifiers. And produced an accuracy of $85.19 \%$, using SVM classifier.The experimental results of classification methods for the extracted features. SVM predicts Recall of $(50 \%)$, accuracy of $(85.19 \%)$ and F1_scoreof $(46 \%)$ andNaïve Bays classification predicts Precision of $(45.62 \%)$.

[10] 10 types ofskin lesions were classified using linear classifier. The last layer of AlexNet was replaced with a convolutional layer. Feature extraction was also performed using an AlexNet. Authors usedthe public Dermo fit Image Library, which has 1300 clinical images, and the above slightly modified AlexNet was tested using those images. 10 types of skin lesionswere present in entire dataset of 1300 clinical images. The accuracy that was achieved on entire dataset which has 10 different types of skin lesions was $81.8 \%$.

[11] Used vector-based SURF approach for the recognition of lesion pattern. The features found are classified using multi SVM classifier to classify the type of lesion.This system provided $86.37 \%$ accuracy, $86.53 \%$ sensitivity and $96.42 \%$ specificity rates. 611 data images were usedwhich has 4 types of skin lesion classes.

[16] proposed computerized method which is fully automatic for skin lesion classification. In this research they pre trained three models ResNet-18, AlexNet, and VGG16 as feature generators. Support Vector Machines are then trained using these extracted features. In the last stage, these classifier outputs are fused to obtain classification. They used 150 images from ISIC 2017, yielding a performance of $83.83 \%$ for melanoma and $97.55 \%$ for seborrheic keratosis classification.

4.2 Skin Lesion Classification Using Convolutional Neural Network as End-to-End Learning 


\subsection{2.a. Transfer learning using a CNN}

[13] Did the ISIC 2018 challenge.They used the HAM10000 Dataset [14]. They used 10015 images for training and validation. $80 \%$ of the images were used to train and $20 \%$ for validation. 6705 of the total number of images were Melanocytic nevus and small category dermatofibroma has 115 images, and some of the remaining images are melanoma, and the rest are of other types.They trained variety of CNN models DenseNet201, ResNet152, Inception _V4. They pretrained their models from ImageNet dataset.For Melanocytic nevus they achieved a confusion matrix of 0.96,0.96,0.96 65 with DenseNet201, ResNet152, Inception V4 respectively. For dermatofibroma they achieved a confusion matrix of $0.86,0.94,0.8265$ with DenseNet201, ResNet152, Inception V4 respectively. For melanoma they achieved a confusion matrix of $0.73,0.76,0.65$ with DenseNet201, ResNet152, Inception V4 respectively. They achieved a $2 \%$ improvement in confusion matrix which is of 0.98 for Melanocytic nevus using DenseNet201 by cropping images for training and validation.

[17] Used CNNs which were trained on datasets ImageNet and a network trained on Dermnet-A skin disease atlas. They used a common CNN called AlexNet for classification and they achieved an accuracy of $89.3 \%$ with a sensitivity of $77.1 \%$ and a specificity of $93.0 \%$.

[18] used a CNN trained with 129,450 images, 3374 of the total images were taken from dermatoscopic devices which represents 2032 different types of skin lesions. malignant melanomas vs benign nevi and keratinocyte carcinomas vs benign seborrheic keratosis were addressed in this study. They used GoogleNet Inception v3 model for classification. The CNN was then tested with test data and achieved an AUC ROC of0.96 for carcinomas and 0.96 for melanomas and an ROC AUC of 0.94 for melanomas classified with dermatoscopic images.

[21] They proposed new prediction model novel regularizer technique that classifies a given lesion into either benign or malignant. So, this is a binary classifier. The data set is taken from ISIC, 5600 images are used for training CNN, and 2400 images for validation. This proposed model achieved an accuracy of $97.49 \%$ in determining benign vs malignant. The performance of $\mathrm{CNN}$ in terms of AUC-ROC is calculated for different cases with an embedded novel regularizer. AUC(area under the curve) achieved for, seborrheic keratosis vs basal cell carcinoma lesionis 0.93.AUC achieved for nevus against melanoma lesion is 0.77. AUC achieved for solar lentigo vs melanoma lesion and seborrheic keratosis vs melanoma lesion are $0.86,0.85$ respectively.

[23]In this research they applied transfer learning to AlexxNet model in different ways, one of the approach is they replaced the classification layer with a softmax layer, another approach they used is fine tuning the weights of architecture, and the last one being augmenting data set by fixed and random rotation angle. Softmax layer is able to classify segmented color image lesions into nevus, seborrheic keratosis and melanoma. The data set ISIC containing 2000 images of which 374 are Melanoma, 254 Seborrheic Keratosis, 1372 images are Nevus were taken, and from Derm (IS \& Quest) 206 images of skin lesion divided to 87 and 119 images for nevus and melanoma, and from MED-Node 170 total images out of which 70 and 100 images for melanoma and nevus images, are used in testing and verifying the proposed method. Accuracy achieved for ISIC is $95.91 \%$, and for Derm (IS \& Quest) is $97.70 \%$, and for MED-NODE is $96.86 \%$.

[24]The effectiveness and also capability of CNNs have been studies in this research by classifying 8 skin diseases. Pretrained state-of-the-art architectures like InceptionResNet v2, ResNet 152, DenseNet 201, and Inception v3 are used. 10135 dermoscopy images are used ,10015 from HAM 10000 and 120 images from $\mathrm{PH} 2$, and this dataset includes 8 types of skin cancers, basal cell carcinoma, melanoma, actinic keratosis, vascular lesions, melanocytic nevi, benign keratosis, atypical nevi and dermatofibroma. The results proved that they outperformeddermatologists by $11 \%$. The best AUC ROC values for basal cell carcinoma and melanoma are 99.30\% (DenseNet 201 ) and $94.40 \%$ (ResNet 152) compared to $88.82 \%$ and $82.26 \%$ for dermatologists. Andalso, DenseNet 201 had the highest micro and macro AUC averaged values for overall classification, which is $98.79 \%$ and $98.16 \%$ respectively.

[26] The team has made their computer algorithm which they used for this research publicly available. AnResNetmodelwas developed by them and was fine-tuned with 19,398 images for training purposes. They used this developed classifier to classify 12 different types of skin diseases. They used public dataset, Asan for classification using the CNN and achieved .96 for melanoma, .83 for squamous cell carcinoma, 0.96 for basal cell carcinoma, and 0.82 for intraepithelial carcinoma. [25] A deep CNN is trained using 4867 clinical images dataset obtained from University of 
Tsukuba Hospital form 2003 until 2016, from 1842 patients diagnosed with skin cancer and tumors. These images consist of 14 Malignant and benign conditions. This research was performed against 13 certified dermatologists and 9 dermatology trainees. The accuracy percentage that was achieved for classification using trained DCNN was 76.5\%. DCNN achieved $89.5 \%$ specificity and $96.3 \%$ sensitivity. And the conclusion is DCNNN classified skin lesion more accurately compared to board certified dermatologists.

[29] Authors have used convolutional neural network with fisher vector encoding and SVM classifier. They eliminated small dataset problems by giving samples or sub-images as the input, instead of whole images as input to the CNN. 1279 skin images from ISBI 2016 dataset was used, the proposed method achieved an accuracy of $83.09 \%$.

\subsection{2.b. Learning from scratch using a $C N N$}

[27] Classification of skin lesion of melanoma Versus nevi or lentigines using dermatoscopic images was done by authors in this research using VGGNet. They compared different techniques of learnings, pretrained CNN using transfer learning and frozen layers versus a CNN trained from scratch. It is one of the papers we found, that did use CNN classification from scratch.

[35] A CNN of Two-layers was trained from scratch for the distinction of benign nevi versus melanoma based on clinical images. They trained the model with 136 images. and 34 images were used as test data. The images are taken from public the University Medical Center Groningen Dermatology Department. They achieved a sensitivity of $81 \%$, a specificity of $80 \%$, and an accuracy of $81 \%$ with this method. As the data taken for training and testing were limited the result should be viewed critically.

\section{Skin Lesions Classification based on Convolution Neural Networks: Vulnerabilities}

We found some research papers that did research on vulnerabilities in using $\mathrm{CNN}$ for skin lesion classification, below are our review on those research papers.

CNNs are proved effective for classifying and analyzing skin cancer diagnosis. But there are limitations of the architecture of CNN in the ability to classify images sometimes. Incorrect classification so skin cancer as benign can cause serious consequences and therefore we should have full understanding of potential failures modes for CNN classifier.

[31] CNNs can be misled into incorrect classification by artificially perturbing natural-world images. This type of manipulation of an input image with the goal of deceiving the network into an incorrect classification is called an 'adversarial attack'[31]. In this paper we will discuss some of these adversarial attacks could arise accidentally in clinical settings and this is from research paper with proper data and findings [31].

- Alterations in color balance

- Alterations in rotation/translation of the input image that lead to misclassification of melanoma as a benign naevus

Authors implemented a CNN for melanoma versus benign melanocytic naevi classification. Theyfine-tuned Inception v3 which is pretrained on the data of skin lesion images from ISIC 2018 dataset. They implemented FGSM attack which makes adjustments to blue green and red values for each pixel in the input image according to magnitude of the pixel, and this will affect the final classification by incorrectly classifying [34]. Second attack they did was 3-pixel attack, by modifying only 3 pixels in the image and leaving other unchanged. They found that this also led to successful attack.CNNs are usually trained with images from dermoscopy. The color balance is influenced by skin pigmentation, image capture, illumination and processing. They tested to see if the image color will influence the accuracy of skin lesion classification, and they figured that numerous number of melanoma images with alterations in RGB colors led to misclassification as benign naevus. They also tried to see if it could be mitigated, by training CNN with varying the image colors and they found 33\% decrease in adversarial attack rate. Next, they tested the images with some more variation, and by subtracting 10 units from green channel led to $235 \%$ increase in false negative for melanoma diagnosis.

Second test is, they tested on rotation of images, whether that could impact the correctness in classifying image. They applied evolution-based optimization method, by allowing arbitrary combinations of rotation up to 360 degrees and translation up to 50 pixels input image size 299x299 pixels in both horizontal and vertical directions. And they found that $45.6 \%$ of images used for testing, deceived classifier into classifying 
melanoma as benign naevus with just rotation and translation of image. They also tested the images by 45 degree and 180-degree rotation and both cases increased false negative rate by $11 \%$.

[32] In a recent study, a publication reported that the presence of blue marker ink in dermoscopic images also had negative impact on CNN classification accuracy.

[33] A substantial difference in skin classification result has been identified by a real world study of CNN accuracy in classifying skin cancer, and according to this study it made a difference in how the images are taken, whether it is taken by iPhone, Samsung or DSLR, all gave different results.

\section{RESULTS AND DISCUSSION}

From the studies that are reviewed,CNN was the best in performing classification of all other architectures. The winner of Large-Scale Visual Recognition Competition (ILSVRC) in 2012 was AlexNet and in 2015 it was, AlexNet, GoogleNet, ResNet and VGGNet. [10,16,17] used AlexNet to perform skin classification and the accuracy was $81.8 \%$ for [10] vs $83.83 \%$ for [16] vs $89.3 \%$ for [17]. When you compare results of [10] and [16], the possibility that [16] achieved better accuracy is because they only took 150 images to test compared to [10] of 1300 images of 10 skin lesion types.

[24] and [26] used ResNet150 to perform the skin lesion classification. This is one of the ideal situations to compare because of the fact that, both used same type of architecture, and also they used large number of images to perform this classification. [24] used 10015 images from HAM 10000 and 120 images from PH2, and [26] used 19,398 of images. Both authors used images form public database, and the accuracy obtained by [24] is $98.16 \%$, and by [26] is $96 \%$. [24] has 8 different types of skin lesions in their images, and [26] has 12 different types of skin lesions.

Another importantchallenge in this research is using archives that mainly contain skin lesions from light skinned people. For example, the images of ISIC, is mainly from United States, Australia, and Europe. To also achieve accurate results of classification for dark skinned people, CNN must be trained to abstract from different skin colors as well. And this can be achieved by taking into consideration of dark-skinned images. Improvement can be made in classification quality by adding clinical data of different age, size of the image, gender, skin type, as inputs for the classifiers.

\section{CONCLUSSION}

We have reviewed multiple studies that does skin lesion classification based on images, we compared some of the methods used by few authors, and the others were difficult to compare because of difference in approaches they have used, and data amount they used. Future publications should use publicly available benchmarks and fully disclose methods used for comparability purposes. Also few negative factors also need to be considered and researched like vulnerabilities of adversarial attacks, and instead of taking all the positive data which will make the system biased and give out only positive result, if we take negative data too it will give accurate results( same goes with vice versa).It would be helpful if further publications take this into consideration, to see the accuracy of $\mathrm{CNN}$ in performing skin lesion classification.

\section{REFERENCES:}

[1] Nami N, Giannini E, Burroni M, Fimiani M, Rubegni P Teledermatology: State-of-the-art and future perspectives. Expert Rev Dermatol. 2014 Jan 10;7(1):1-3. doi: 10.1586/edm.11.79. [CrossRef] [Google Scholar]

[2] Fabbrocini G, Triassi M, Mauriello MC, Torre G, Annunziata MC, De Vita V, Pastore F, D'Arco V, Monfrecola G. Epidemiology of skin cancer: Role of some environmental factors. Cancers (Basel) 2010 Nov 24;2(4):1980-1989.

10.3390/cancers2041980. http://www.mdpi.com/resolve r?pii=cancers2041980. [PMC free article] [PubMed] [CrossRef] [Google Scholar]

[3] Argenziano G, Soyer HP. Dermoscopy of pigmented skin lesions: A valuable tool for early diagnosis of melanoma. Lancet Jul;2(7):443-449. [PubMed] [Google Scholar]

[4] Kittler H, Pehamberger H, Wolff K, Binder M. Diagnostic accuracy of dermoscopy. Lancet Oncol. 2002 Mar;3(3):159-165. [PubMed] [Google Scholar]

[5] Ali ARA, Deserno TM. A systematic review of automated melanoma detection in dermatoscopic images and its ground truth data. Proc SPIE Int Soc Opt Eng. 2012 Feb 28;8318:1-6. doi: 10.1117/12.912389. [CrossRef] [Google Scholar].

[6] Fabbrocini G, De Vita V, Pastore F, D'Arco V, Mazzella C, Annunziata MC, Cacciapuoti S, Mauriello MC, Monfrecola A. Teledermatology: From prevention to diagnosis of nonmelanoma and melanoma skin cancer. Int $\mathrm{J}$ Telemed Appl. 2011 Sep 01;2011(17):125762. doi: 10.1155/2011/125762. doi: 10.1155/2011/125762. [PMC free article] [PubMed] [CrossRef] [CrossRef] [Google Scholar]

[7] LeCun Y, Bengio Y, Hinton G. Deep learning. Nature. 2015 May 28;521(7553):436-444. doi: 10.1038/nature14539. [PubMed] [CrossRef] [Google Scholar]

[8] Krizhevsky A, Sutskever I, Hinton GE. ImageNet classification with deep convolutional neural 
networks. Adv Neural Inf Process Syst. 2012 Jan;:1-23. doi: 10.1145/3065386. [CrossRef] [Google Scholar]

[9] Seeja R.D., Suresh A. Deep Learning Based Skin Lesion Segmentation and Classification of Melanoma Using Support Vector Machine (SVM) Asian Pac. J. Cancer Prev. 2019;20:1555-1561.

10.31557/APJCP.2019.20.5.1555. [PMC

doi: article] [PubMed] [CrossRef] [Google Scholar]

[10] Kawahara J, BenTaieb A, Hamarneh G. Deep features to classify skin lesions. Proceedings of the 2016 IEEE 13th International Symposium on Biomedical Imaging (ISBI); 2016 IEEE 13th International Symposium on Biomedical Imaging (ISBI); April 13-16, 2016; Prague, Czech Republic. 2016. [CrossRef] [Google Scholar]

[11] Thompson F, Jeyakumar MK. Vector based classification of dermoscopic images using SURF. IJAER. 2017;12:1758-64. [Google Scholar]

[12] Pomponiu V, Nejati H, Cheung NM. Deepmole: Deep neural networks for skin mole lesion classification. Proceedings of the 2016 IEEE International Conference on Image Processing (ICIP); 2016 IEEE International Conference on Image Processing (ICIP); September 25-28, 2016; Phoenix, AZ. 2016. [Google Scholar]

[13] K. M. Li and E. C. Li. Skin lesion analysis towards melanoma detection via end-to-end deep learning of convolutional neural networks. CoRR, abs/1807.08332, 2018.

[14] Tschandl, P., Rosendahl, C., Kittler, H.: The ham10000 dataset: A large collection of multi-sourcedermatoscopic images of common pigmented skin lesions. arXiv preprint arXiv: 1803.10417 (2018)

[15] M. Vestergaard, P. Macaskill, P. Holt, S. MenziesDermosc opy compared with naked eye examination for the diagnosis of primary melanoma: a meta-analysis of studies performed in a clinical setting $\mathrm{Br} J$ Dermatol, 159 (3) (2008), pp. 669-676

[16] A. Mahbod, R. Ecker, and I. Ellinger. (Feb. 2017). "Skin lesion classification using hybrid deep neural networks." [Online]. Available:https://arxiv.org/abs/1702.08434v1

[17] A. Nylund, "To be, or not to be Melanoma: Convolutional neural networks in skin lesion classification," Ph.D. dissertation, School Technol. Health, KTH Roy. Inst. Technol., Stockholm, Sweden, 2016. [Online]. Available: http://kth.diva-portal.org/smash/get/diva2:950147/FUL LTEXT01.pdf

[18] A. Esteva et al., "Dermatologist-level classification of skin cancer with deep neural networks," Nature, vol. 542, no. 7639, pp. 115-118, 2017.

[19] A. A. Ali and H. Al-Marzouqi, "Melanoma detection using regular convolutional neural networks," in Proc. Int. Conf. Elect. Comput. Technol. Appl. (ICECTA), Nov. 2017, pp. $1-5$.

[20] M. Rehman, S. H. Khan, S. M. D. Rizvi, Z. Abbas, and A. Zafar, "Classification of skin lesion by interference of segmentation and convolotion neural network," in Proc. 2nd Int. Conf. Eng. Innov. (ICEI), Jul. 2018, pp. 81-84.

[21] Albahar, M. A., Skin lesion classification using convolutional neural network with novel Regularizer. IEEE Access 7:38306-38313, 2019.

[22] A. Demir, F. Yilmaz and O. Kose, "Early detection of skin cancer using deep learning architectures: resnet-101 and inception-v3," 2019 Medical Technologies Congress (TIPTEKNO), Izmir, Turkey, 2019, pp. 1-4, doi: 10.1109/TIPTEKNO47231.2019.8972045.

[23] Hosny KM, Kassem MA, Foaud MM (2019) Classification of skin lesions using transfer learning and augmentation with
Alex-net. PLoS ONE 14(5): e0217293. https://doi.org/10.1371/journal.pone.0217293

[24] Rezvantalab, Amirreza, Habib Safigholi, and Somayeh Karimijeshni. "Dermatologist level dermoscopy skin cancer classification using different deep learning convolutional neural networks algorithms." arXiv preprint arXiv: 1810.10348 (2018).

[25] Fujisawa, Y., Y. Otomo, Y. Ogata, Y. Nakamura, R. Fujita, Y. Ishitsuka, R. Watanabe, N. Okiyama, K. Ohara, and M. Fujimoto. "Deep-learning-based, computer-aided classifier developed with a small dataset of clinical images surpasses board-certified dermatologists in skin tumour diagnosis." British Journal of Dermatology 180, no. 2 (2019): 373-381.

[26] Han SS, Kim MS, Lim W, Park GH, Park I, Chang SE. Classification of the clinical images for benign and malignant cutaneous tumors using a deep learning algorithm. J Invest Dermatol 2018 Jul;138(7):1529-1538. [CrossRef] [Medline]

[27] Lopez AR, Giro-i-Nieto X, Burdick J, Marques O. Skin lesion classification from dermoscopic images using deep learning techniques. Proceedings of the 13th IASTED International Conference on Biomedical Engineering; 13th IASTED International Conference on Biomedical Engineering; February 20-21, 2017; Innsbruck, Austria. 2017. [CrossRef] [Google Scholar]

[28] Cerwall, P. \& Report, E. M. Ericssons mobility report https://www.ericsson.com/ res/docs/2016/ericsson-mobility-report-2016.pdf (2016).

[29] Yu, Z., Ni, D., Chen, S., Qin, J., Li, S., Wang, T., Lei, B.: Hybrid dermoscopy image classification framework based on deep convolutional neural network and fisher vector. In: Biomedical Imaging (ISBI 2017), 2017 IEEE 14th International Symposium on, IEEE (2017) 301-304

[30] https://towardsdatascience.com/confusion-matrix-for-yo ur-multi-class-machine-learning-model-ff9aa3bf7826

[31] Du-Harpur, Xinyi, Callum Arthurs, Clarisse Ganier, Rick Woolf, Zainab Laftah, Manpreet Lakhan, Amr Salam et al. "Clinically-relevant vulnerabilities of deep machine learning systems for skin cancer diagnosis." The Journal of Investigative Dermatology (2020).

[32] Winkler JK, Fink C, Toberer F, Enk A, Deinlein T, Hofmann-Wellenhof R, et al. Association Between Surgical Skin Markings in Dermoscopic Images and Diagnostic Performance of a Deep Learning Convolutional Neural Network for Melanoma Recognition [Internet]. JAMA Dermatology. 2019. Available from: http://dx.doi.org/10.1001/jamadermatol.2019.1735

[33] Phillips M, Marsden H, Jaffe W, Matin RN, Wali GN, Greenhalgh J, et al. Assessment of Accuracy of an Artificial Intelligence Algorithm to Detect Melanoma in Images of Skin Lesions. JAMA Netw Open. 2019;2(10):e1913436

[34] Goodfellow IJ, Shlens J, Szegedy C. Explaining and Harnessing Adversarial Examples [Internet]. arXiv [stat.ML]. 2014. Available from: http://arxiv.org/abs/1412.6572

[35] Nasr-Esfahani E, Samavi S, Karimi N, Soroushmehr SMR, Jafari MH, Ward K, Najarian K. Melanoma detection by analysis of clinical images using convolutional neural network. Proceedings of the 38th Annual International Conference of the IEEE Engineering in Medicine and Biology Society (EMBC); 38th Annual International Conference of the IEEE Engineering in Medicine and Biology Society (EMBC); August 16-20, 2016; Orlando, FL. 2016. [Google Scholar]

[36] Brinker, Titus Josef, Achim Hekler, Jochen Sven Utikal, Niels Grabe, Dirk Schadendorf, Joachim Klode, Carola 
Berking, Theresa Steeb, Alexander H. Enk, and Christof von Kalle. "Skin cancer classification using convolutional neural networks: systematic review." Journal of medical Internet research 20, no. 10 (2018): e11936.

[37] Masood, Ammara, and Adel Ali Al-Jumaily. "Computer aided diagnostic support system for skin cancer: a review of techniques and algorithms." International journal of biomedical imaging 2013 (2013).

[38] Rezvantalab, Amirreza, Habib Safigholi, and Somayeh Karimijeshni. "Dermatologist level dermoscopy skin cancer classification using different deep learning convolutional neural networks algorithms." arXiv preprint arXiv: 1810.10348 (2018).

[39] Amin, Javeria, Abida Sharif, Nadia Gul, Muhammad Almas Anjum, Muhammad Wasif Nisar, Faisal Azam, and Syed Ahmad Chan Bukhari. "Integrated design of deep features fusion for localization and classification of skin cancer." Pattern Recognition Letters 131 (2020): 63-70.

[40] Mehta, Palak, and Bhumika Shah. "Review on techniques and steps of computer aided skin cancer diagnosis." Procedia Computer Science 85 (2016): 309-316.

[41] Siegel, Rebecca L., Kimberly D. Miller, and Ahmedin Jemal. "Cancer statistics, 2015." CA: a cancer journal for clinicians 65, no. 1 (2015): 5 .

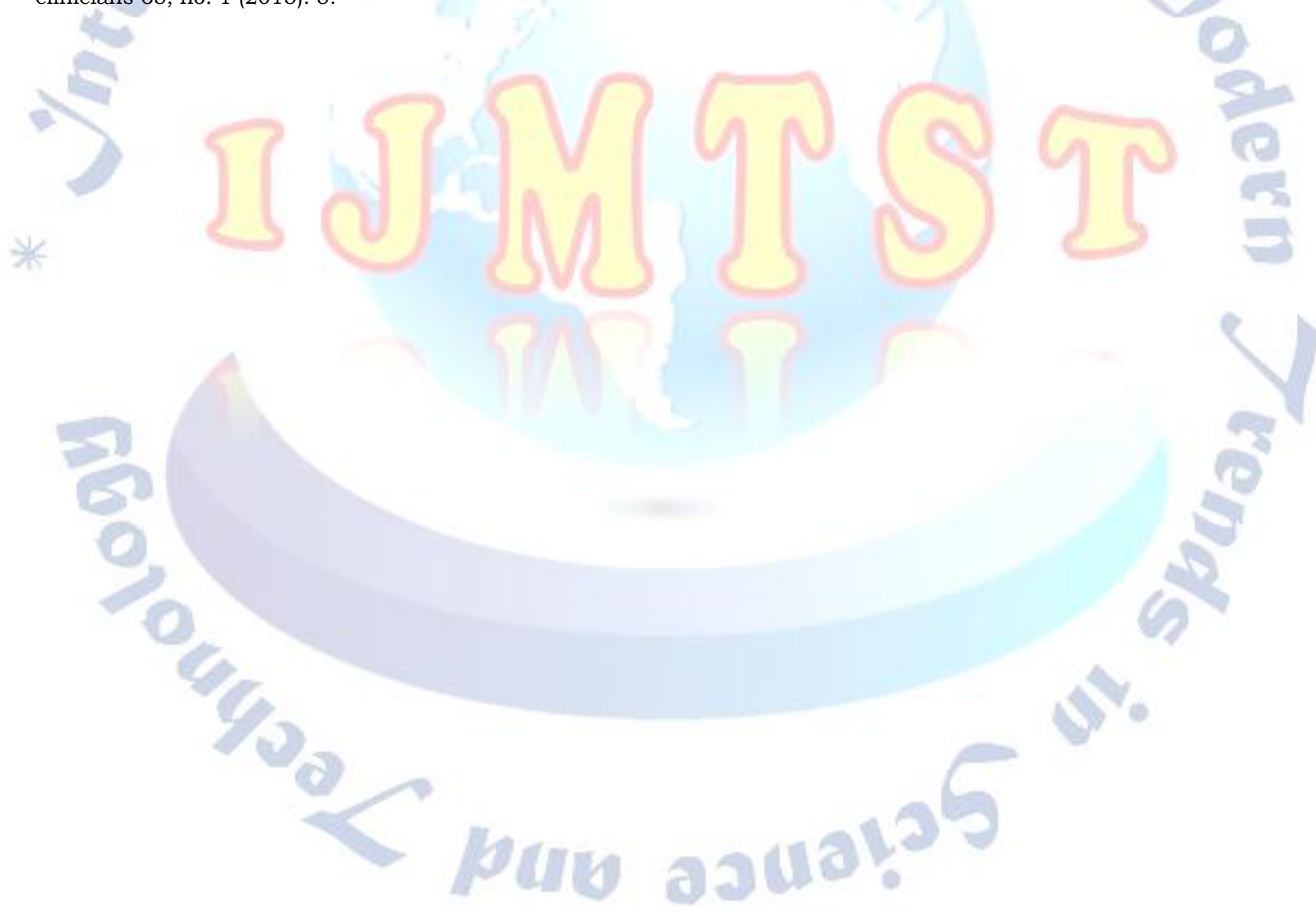

